\title{
Lobar Distribution of Low Grade Oligodendroglioma: Distribution, Molecular Characteristics, and Survival Based upon Location
}

\author{
Donald A. Ross*, Shao Tao, Sakir Gultekin, Amy M. Ross \\ Portland Veterans Medical Center, Oregon Health \& Science University, Portland, Oregon, USA \\ Email: ${ }^{*}$ rossdo@ohsu.edu
}

Received 21 August 2014; revised 18 September 2014; accepted 13 October 2014

Academic Editor: Sibu P. Saha, University of Kentucky, USA

Copyright (C) 2014 by authors and Scientific Research Publishing Inc.

This work is licensed under the Creative Commons Attribution International License (CC BY). http://creativecommons.org/licenses/by/4.0/

c) (i) Open Access

\section{Abstract}

Grade II oligodendrogliomas are rare and slow growing tumors, making long-term follow up difficult, but necessary for better understanding. In this retrospective study a review of all grade II oligodendrogliomas encountered in the last 20 years at one institution, was undertaken to determine if specific tumor location and immunohistochemical analysis had any impact on recurrence rate, progression free survival, or life expectancy. Eighty-nine grade II oligodendroglioms cases were reviewed ( 38 females and 51 males; mean age was $40.3 \pm 13.8$ years). Tumor location was: frontal lobe $(44,49.4 \%)$ and superior frontal gyrus $(30,33.7 \%) .1 \mathrm{p} 19 \mathrm{q}$ data were available in 49 patients. Twenty-nine cases were co-deleted (59.2\%). There was no significant difference in the incidence of $1 \mathrm{p} 19 \mathrm{q}$ co-deletion between superior frontal gyrus tumors vs. other frontal tumors or extra-frontal tumors $(p=0.45)$. Follow up of at least 3 months after diagnosis was available in 79 patients (mean follow up: 93.2 months). In recurrence analysis, recurrence by $1 \mathrm{p} 19 \mathrm{q}$ status and recurrence by location revealed no significant differences. In analysis of progression, progression by 1p19q status and progression by location revealed no significant differences. An analysis of deaths for the sample, deaths by $1 \mathrm{p} 19 \mathrm{q}$ status and deaths by location revealed no significant differences. There was a higher death rate among patients $>50$ years of age, however this, too, was not significant. There did not appear to be any advantage in recurrence rate, progression free survival, or life expectancy for tumors located in the frontal lobe or superior frontal gyrus. 1p19q co-deletion did not appear to confer an advantage as measured by time to recurrence, time to progression, or overall survival. Other than age, eloquent location, Karnofsky status, and overall tumor size as reported by others, tumor location and $1 \mathrm{p} 19 \mathrm{q}$ status in low grade oligodendrogli-

\footnotetext{
${ }^{*}$ Corresponding author.
}

How to cite this paper: Ross, D.A., Tao, S., Gultekin, S. and Ross, A.M. (2014) Lobar Distribution of Low Grade Oligodendroglioma: Distribution, Molecular Characteristics, and Survival Based upon Location. Journal of Cancer Therapy, 5, 12491258. http://dx.doi.org/10.4236/jct.2014.513126 
omas are not currently predictive of survival.

\title{
Keywords
}

\author{
Grade II Oligodendrogliomas, Frontal Lobe, Superior Frontal Gyrus, 1p19q, Isocitrate \\ Dehydrogenase 1
}

\section{Introduction}

The senior author's personal experience with World Health Organization (WHO) grade II oligodendrogliomas [1] showed that 10 of 19 patients had tumors in the frontal lobe and four patients were alive and event free $>10$ years after diagnosis, three of whom had tumors in the superior frontal gyrus. To test the hypotheses that oligodendrogliomas were more common in the frontal lobe and that such tumors confer an improved prognosis, a review of all such tumors encountered at Oregon Health \& Science University (OHSU) over the last 20 years was conducted.

\section{Materials and Methods}

\subsection{Clinical Data}

With the approval of the OHSU Institutional Review Board, pathology department records were surveyed for all specimens diagnosed as oligodendrogliomas by WHO criteria [2]. Electronic patient medical charts and imaging data were reviewed for age and gender, date of diagnosis, lobar location of the tumor on magnetic resonance imaging (MRI), pathology reports, molecular pathology data, treatments rendered, recurrence, overall survival, and progression free survival. The authors reviewed patients with WHO grade II oligodendrogliomas as confirmed by the neuropathology staff at OHSU according to standards available at the time of diagnosis. All patients were jointly managed at a multi-disciplinary Brain Tumor Board, but all patients were treated on an individual basis and not based upon a protocol.

\subsection{Immunohistochemistry}

Isocitrate dehydrogenase 1 (IDH1) analysis was performed as follows: DNA was extracted from tumor-rich areas of formalin-fixed paraffin-embedded (FFPE) specimens using the Macherey-Nagel Nucleospin kit (Macherey-Nagel Inc., Bethlehem, PA). Mutations in IDH1 exon 2 and IDH2 exon 4 were detected by high resolution melting curve analysis on a Roche Light Cycler 480 instrument (Roche Diagnostics, Indianapolis, IN), using LC Green Plus Dye (Idaho Technology, Salt Lake City, UT). Specimens with a melt profile that deviated from wild type were subjected to Sanger sequence analysis to identify the specific mutation. The estimated sensitivity of this method is approximately $20 \%$ mutant allele.

Fluorescent in situ hybridization (FISH) on paraffin sections was performed by the OHSU Knight Diagnostic Laboratories Clinical Cytogenetics Laboratory. Deparaffinization and pretreatment were performed using a VP2000 processor (Abbott Laboratories, Abbott Park, IL). Abbott probes for chromosome 1p36 (1q25 control) and chromosome 19q13 (19p13 control) were used. Hybridization, washing, and counterstaining were performed according to the probe manufacturer's instructions. 100 interphase cells were scored for each probe. The ratio of the total number of test probe signals to the total number of control probe signals was calculated. Deletion was defined as a ratio of $<0.80$ for both probe sets. Fluorescence was visualized on a CytoVision image capture system (Applied Imaging, San Jose, CA) with a Nikon E800 (Nikon, Melville, NY) fluorescence microscope with appropriate filters suggested by the probe manufacturer.

\subsection{Statistical Analysis}

Chi square analysis and analysis of variance with the Bonferroni correction were performed using Stata/IC 12.1 (Stata Corp., College Station, Texas) and data recorded as \pm standard deviation (SD) and interquartile range (IQR). 


\section{Results}

Eighty-nine patients meeting criteria were reviewed (Table 1). There were 38 females and 51 males. Ages ranged from 8 to 73 years (mean $40.3 \pm 13.8$; median 41, and IQR 43.5 - 48.5).

\subsection{Tumor Location}

All 89 tumors were localized radiographically. Thirty (68.2\%) of 44 frontal lobe tumors and 30 (33.7\%) of 89 total were located in the superior frontal gyrus (SFG). Mean age of patients with tumors in the SFG, for any frontal tumor, and for tumors extra-frontal lobe or multi-lobar was 39.3, 38.8 and 41.7 years, respectively. Chi square analysis revealed significant differences between men and women and laterality; men having equal numbers of tumors in the left and right hemispheres (25 vs. 26) and women having more tumors in the left than in the right hemisphere (26 vs. $11 ; p=0.046)$, there was no significant difference between genders and location $(p=$ 0.82). Chi square analysis revealed no significance difference between laterality and location $(p=0.26)$. Analysis of variance with the Bonferroni Correction was not significant between age and location $(p=0.41)$.

Table 1. Demographics and clinical characteristics $(n=89)$.

\begin{tabular}{|c|c|}
\hline Demographic & \\
\hline Age (years) mean $( \pm S D)$; range & 40.3 (13.8); 8 - 73 \\
\hline \multicolumn{2}{|l|}{ Gender; n (\%) } \\
\hline Male & $51(57)$ \\
\hline Female & $38(43)$ \\
\hline \multicolumn{2}{|l|}{ Clinical characteristic } \\
\hline \multicolumn{2}{|l|}{ Location; n (\%) } \\
\hline Right hemisphere & $37(42)$ \\
\hline Left hemisphere & $51(57)$ \\
\hline Data missing & 1 \\
\hline Frontal & $44(49)$ \\
\hline Superior frontal gyrus & 30 \\
\hline Middle, inferior, or basal frontal gyrus & 14 \\
\hline Extra-frontal & $31(35)$ \\
\hline Temporal lobe & 23 \\
\hline Parietal lobe & 7 \\
\hline Occipital lobe & 1 \\
\hline Multi-lobar & $14(16)$ \\
\hline Fronto-temporal & 7 \\
\hline Temporo-parietal & 6 \\
\hline Fronto-parietal & 1 \\
\hline \multicolumn{2}{|l|}{ Lateralized location; n (\%) } \\
\hline \multicolumn{2}{|l|}{ Superior frontal gyrus } \\
\hline Right & $14(16)$ \\
\hline Left & $16(18)$ \\
\hline \multicolumn{2}{|l|}{ Extra-superior frontal gyrus } \\
\hline Right & $23(26)$ \\
\hline Left & 35 (39) \\
\hline Could not be determined & 1 \\
\hline
\end{tabular}




\subsection{1p19q Co-Deletion}

1p19q data was available in 49 patients. Twenty-nine were co-deleted (59.2\%) and 20 were not, of whom 2 were 1p mono-deleted and 3 were 19q mono-deleted. Twenty-nine patients with tumors in the frontal lobe were tested; 19 (65.5\%) were found to be co-deleted. In 20 patients with tumors extra-frontal lobe or multi-lobar who were tested, 10 (50\%) were co-deleted. In 21 tumors tested in the SFG, both loci were deleted in 13 (61.9\%) patients and $19 q$ in only 1 patient. Chi square analysis revealed no significant difference in the incidence of 1p19q codeletion between SFG tumors vs. other frontal tumors or extra-frontal tumors $(p=0.45)$. Chi-square analysis comparing all 29 tested frontal lobe tumors with all 20 tested extra-frontal tumors revealed no significant difference in $1 \mathrm{p} 19 \mathrm{q}$ deletion $(p=0.277)$.

\subsection{Isocitrate Dehydrogenase 1}

IDH1 data was available in 13 patients. Mutations were found in 9 (69.2\%) patients. In 9 tested patients with frontal lobe tumors, 7 (77.8\%) stained for mutation. In 7 tested patients with tumors in the SFG, 5 stained for mutation (71.4\%). In 4 tested patients with tumors outside the frontal lobe, 2 patients stained for mutation (50\%). Chi squared analysis comparing 7 tested SFG tumors, 2 other frontal tested tumors, and 4 extra-frontal tested tumors revealed no significant difference between the 3 groups $(p=0.45)$. Chi square analysis comparing the 9 tested frontal tumors with the 4 tested extra-frontal tumors revealed no significance difference in IDH1 mutation $(p=0.317)$. Of the 9 patients with IDH1 mutations, 1 with an SFG tumor died 190 months after diagnosis, and 8 were alive an average of 82 months after diagnosis. There was no recurrence, progression or death in the 4 patients without the IDH1 mutation. Therefore, Kaplan Meier Survival analysis was not conducted as there was no information about one of the 2 groups to be entered into Cox Hazards Ratio testing and Kaplan Meier curve graphing.

\subsection{Survival and Recurrence}

Follow up of at least 3 months after diagnosis was available in 79 patients. (9 had undergone neuropathological review at our institution, but had their treatment elsewhere. One patient died 2 months after diagnosis). Mean duration of follow up for the entire series was 93.2 months (88.6 months in 39 patients with tumors outside the frontal lobe and 97.7 months in 40 patients with tumors in the frontal lobe). An analysis of variance showed no difference in overall follow up time between all frontal and all extra-frontal locations $(p=0.51)$.

Documentation of date of recurrence was available in 47 patients at an average of $67.6 \pm 43.2$ months and a median of 60 months, IQR 36 - 91 months after diagnosis (Figure 1). Seventeen recurrences occurred in patients with SFG tumors at a mean of $58.9 \pm 33.6$ months. Five recurrences occurred in patients with other frontal locations at a mean of $97.2 \pm 72.8$ months after diagnosis. Twenty-five recurrences occurred in patients with extra-frontal tumor locations at a mean of $67.6 \pm 41.3$ months. Analysis of variance showed no significant differences between these 3 groups $(p=0.22)$ or between all frontal locations and all extra-frontal locations $(p=$ 0.99).

Mean time to recurrence in patients with 1p19q co-deletion and without 1p19q co-deletion was 72.4 and 76.0 months, respectively (two-tailed t-test; $p=0.83$ ). There were too few patients with IDH1data for statistical testing. Figure 1 shows Kaplan Meier graphs of recurrence, recurrence by 1p19q, recurrence by location, and recurrence by frontal vs. extra-frontal (Figure 1); all hazard ratios (HR) and Harrell's C were not significant.

Progression was defined by change to a higher WHO grade by pathologic analysis at subsequent surgery or by the appearance of new contrast enhancement on MRI in a previously non-enhancing tumor. Progression was determined in 30 patients at a mean time of $87.3 \pm 54.2$ months and a median of 69 months, IQR, 50 - 123 months. Progression occurred in 12 patients with SFG tumors at a mean of $76.5 \pm 40.9$ months after diagnosis. Progression occurred in 4 patients with other frontal lobe locations at a mean of $138 \pm 94.3$ months after diagnosis. Progression occurred in 14 patients with extra-frontal locations at a mean of $82 \pm 46.3$ months after diagnosis. Mean time to progression in patients with $1 \mathrm{p} 19 \mathrm{q}$ co-deletion and without $1 \mathrm{p} 19 \mathrm{q}$ co-deletion was 86.5 and 105.4 months, respectively (two-tailed t-test; $p=0.43$ ). There were too few patients with IDH1data for statistical testing. Figure 2 shows Kaplan Meier graphs of progression, progression by 1p19q, progression by location, and progression by frontal vs. extra-frontal (Figure 2); all HR and Harrell's C were not significant.

Thirty-two of 79 evaluable (40.5\%) patients died an average of $96.7 \pm 60.7$ months, median 100 months, IQR, 

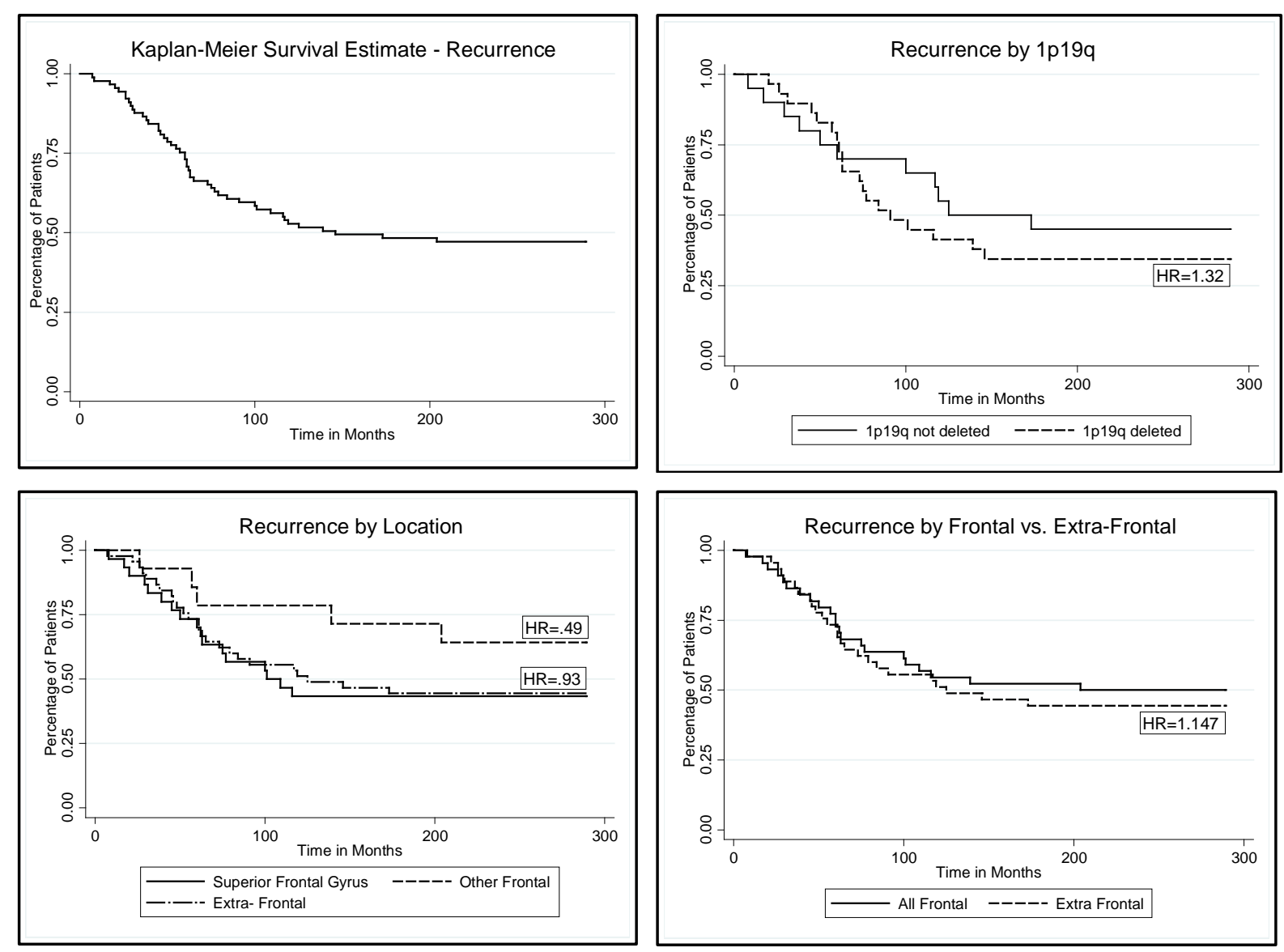

Figure 1. Survival analysis-recurrence by location, frontal vs. extra frontal, 1p19q deletion.

56 - 125 months; range 2 - 289 months after diagnosis. Fifteen patients with frontal lobe tumors died an average of 103.7 months after diagnosis, (if the single patient who died 2 months after diagnosis is excluded, the average is 110.9 months). Seventeen patients with tumors outside of the frontal lobe died an average of 90.5 months after diagnosis. Eleven patients with tumors in the SFG died an average of 99.2 months after diagnosis. Overall survival was $34 \%$ worse for those $>49$ years at diagnosis than those $<50$ years at diagnosis, although not significantly $(\mathrm{HR}=1.34, p=0.50$; Figure 3$)$. Overall survival in patients with $1 \mathrm{p} 19 \mathrm{q}$ co-deletion and without $1 \mathrm{p} 19 \mathrm{q}$ co-deletion was 119 and 110 months, respectively (two-tailed t-test; $p=0.76$ ). There were too few patients with IDH1data for statistical testing. Figure 4 shows Kaplan Meier graphs of deaths for the sample $(\mathrm{n}=79)$, deaths by 1p19q, deaths by location, and deaths by frontal vs. extra-frontal (Figure 4); all HR and Harrell's C were not significant.

Thirteen patients who were tested for 1p19q deletions died at an average of 116.5 months after diagnosis. Nine patients with co-deletions died an average of 114.1 months after diagnosis and 4 patients without co-deletions died an average of 122 months after diagnosis. Only a single patient tested for the IDH1 mutation died at 190 months after diagnosis with a tumor in the SFG, which had tested positive for the mutation. Twenty-five patients survived greater than 119 months. Fourteen had frontal lobe tumors, 11 SFG tumors, and 11 extra-frontal tumors.

\section{Discussion}

Oligodendrogliomas are uncommon primary brain tumors, accounting for about 6\% of gliomas in the Central Brain Tumor Registry of the United States (www.cbtrus.org). The incidence rate for low grade oligodendrogliomas has been estimated to be 0.25 to 0.3 per 100,000 individuals [2] [3]. Relatively long survival compared with glioblastoma means that long-term follow up is necessary to assess the results of treatment. During the 

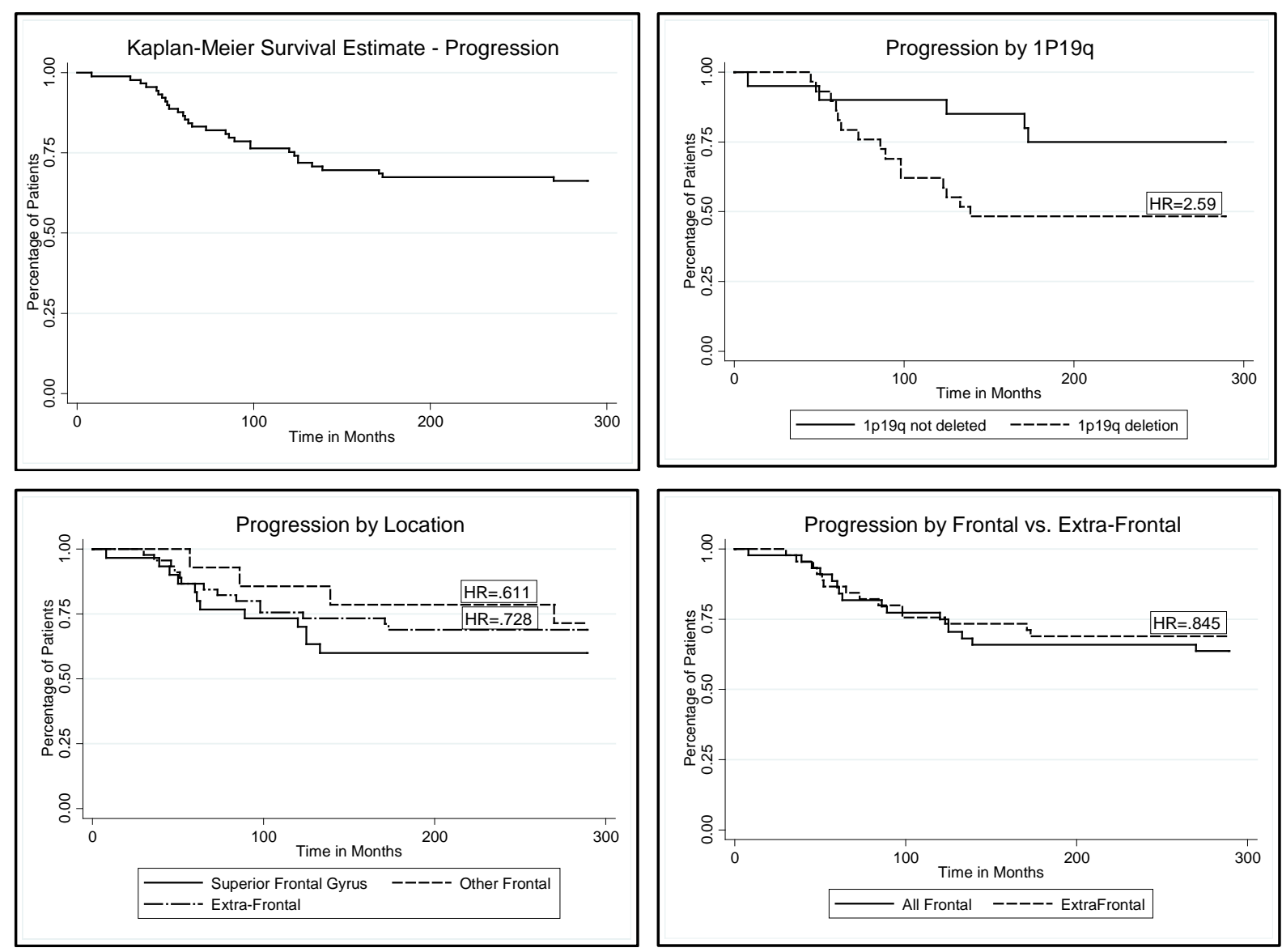

Figure 2. Survival analysis-progression by location, frontal vs. extra frontal, 1p19q deletion.

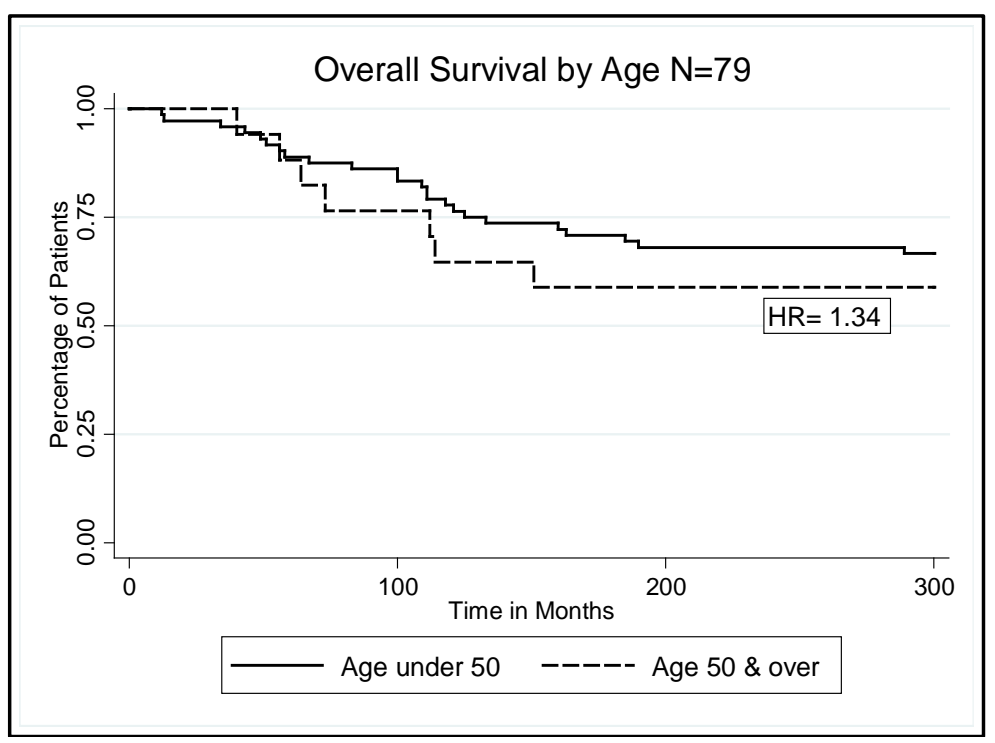

Figure 3. Overall survival by age $>50$ years.

period of follow up of this report, many patients are inevitably lost. In addition, histopathological criteria for diagnosis have changed [2] and molecular definitions of oligodendroglioma have been developed and refined [2] [4]-[6]. Treatment of oligodendrogliomas has evolved considerably [7] [8]. Surgery has changed from 

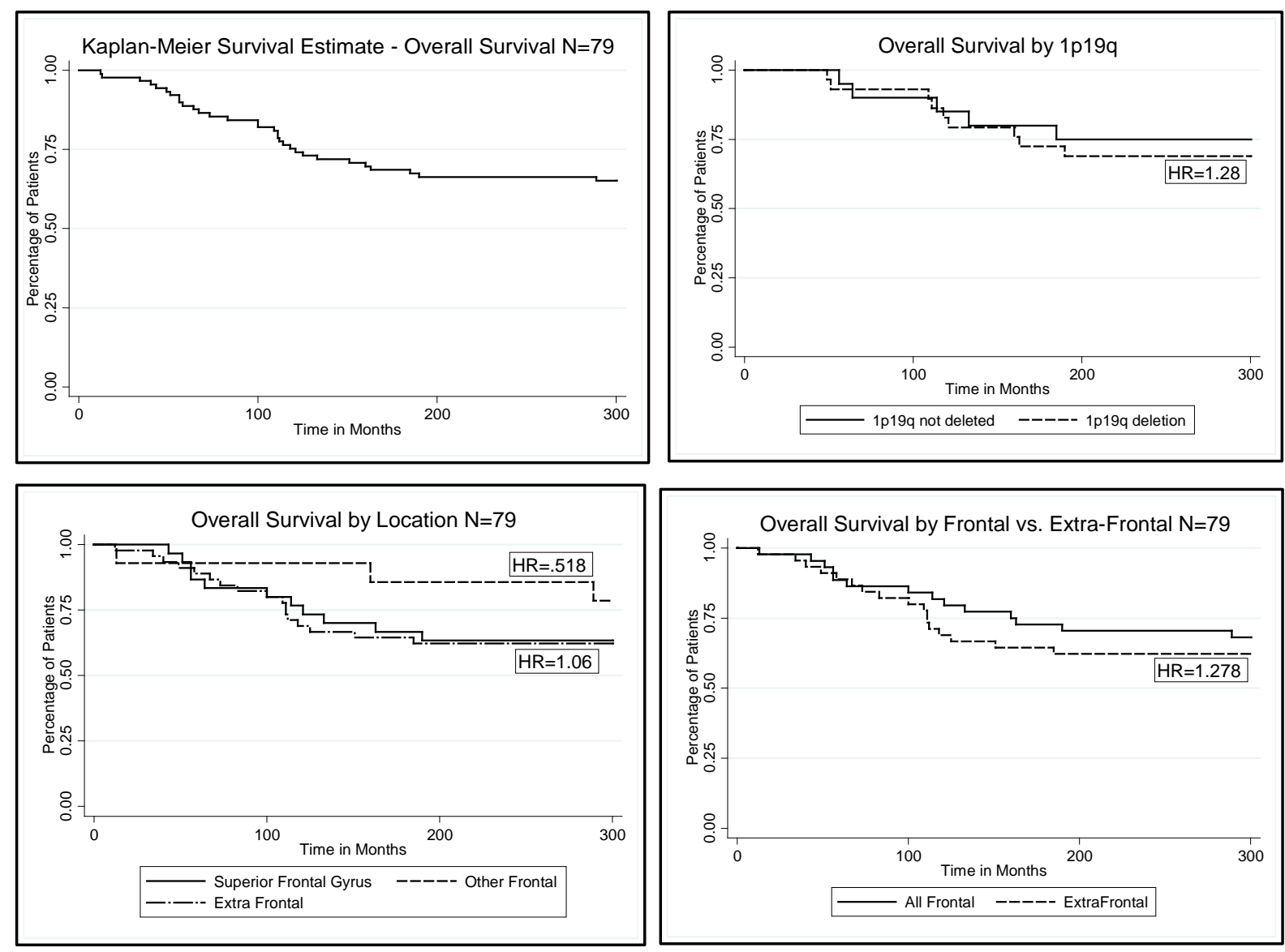

Figure 4. Survival analysis-overall survival by location, frontal vs. extra frontal, 1p19q deletion.

observation or biopsy to aggressive resection with the use of awake craniotomy, cortical and white matter mapping [9], frameless stereotaxy, and better imaging with functional MRI and diffusion tensor imaging tractography [10]. Radiation therapy has changed from large field fractionated treatment to conformal treatment, intensity modulation, and radiosurgery. Chemotherapy has changed from procarbazine, lomustine, and vincristine to temozolamide and bevacizumab. Therefore, it is difficult to describe a uniform group of patients with the same diagnostic criteria and the same treatment protocol. Additionally, previous low grade glioma reports have often included several different tumor types including astrocytomas and mixed gliomas [11]. It is now necessary to move away from these broad categories to report on individual tumor histologies independently [10] [12] [13]. With these limitations in mind, we have endeavored to describe a large group of patients treated at a single institution over 20 years and to investigate the frequency of oligodendrogliomas in different parts of the brain and to determine whether location impacts molecular characteristics or prognosis.

The cerebral cortex is thought to represent $85 \%$ of the brain and the frontal lobes about $41 \%$, with the temporal lobes about 22\%, the parietal lobes 19\%, and the occipital lobes 18\% of the cerebrum [14]. Half (49.4\%) of our series of grade II oligodendrogliomas were in the frontal lobe and one third were in the SFG. If the SFG is a large one of five or more gyri composing the frontal lobe, then approximately $10 \%$ of the cerebrum might be found in this gyrus. Thus, in our series of grade II oligodendrogliomas, the tumors were more common in the frontal lobe and much more common in the SFG than can be accounted for by brain volume alone. In particular, there does appear to be a predilection for the SFG, which accounted for $34 \%$ of the tumors, but only about $10 \%$ of the cortex of the brain.

Other reports have noted a frontal lobe predilection for low grade oligodendrogliomas [8] [15]. In 69 patients from McGill University, 49 (71\%) were noted to be frontal in location and this conferred a prognostic advantage over other locations, but no further localizing information was provided [15]. Another report included 64 patients with anaplastic oligodendrogliomas and reported that 34 (53\%) were predominantly frontal lobe in loca- 
tion [16]. The authors speculated that different locations of oligodendrogliomas may arise from different precursor cells that are region or time specific in the brain or that certain molecular carcinogenic events are only tumorogenic in certain cells or regions of the brain [16].

As multi-variate Cox proportional hazards modeling has been reported to show that location of low grade gliomas in eloquent structures and age greater than 50 years were associated with lower overall survival [17], it might be expected that frontal, non-eloquent locations would convey improved survival. Overall, frontal tumor location in our group was associated with a slight and non-significantly younger age group. We were not able to show a survival advantage for tumors in the frontal lobe, and no advantage in time to recurrence or time to progression in our group. This is similar to the Barrow Neurological Institute report, which noted that extent of resection, presumably more likely to be extensive in frontal locations, did not correlate with the likelihood of malignant progression [10].

1p19q co-deletions have been reported to be present in 39\% - 70\% of oligodendrogliomas [3] [5] and are thought to be relatively homogeneous throughout a tumor [18]. 1p19q deletions have been reported to be more common in frontal oligodendrogliomas than those found elsewhere in the brain [3] [4]. Others have noted that anaplastic oligodendrogliomas located in the temporal lobe, insula, and diencephalon were less likely to harbor 1p19q deletions and responded less well to chemotherapy [16]. One report noted indistinct tumor margins on MRI associated with invasiveness when the co-deletion was present [19], another found that tumors without the co-deletion were more likely to have an infiltrative growth pattern [20]. Imaging pattern on texture analysis has been reported to be highly predictive of $1 \mathrm{p} 19 \mathrm{q}$ status [21], but these reports do not mention a predilection of oligodendrogliomas for specific gyri [20] [21].

Data on tested tumors in this series appear to confirm the reported frontal lobe molecular findings, with an increased likelihood of 1p19q co-deletions in the frontal lobe (65.5\%) versus other lobes (50.0\%) and in the SFG tumors (61.9\%). However, this was not statistically significant and did not appear to confer a survival advantage in patients. Many studies have investigated 1p19q status as a marker for overall survival, with both positive and negative results reported (reviews by Bourne and Schiff, 2010 and Capelle, et al., 2009) [5] [22]. Similarly, predicting response to specific therapeutic interventions has been mixed for WHO grade II oligodendrogliomas, with some prediction of radiation response but less convincing data for chemotherapeutic response [5] [23] [24]. This may differ from recent reports on anaplastic oligodendrogliomas, a report on which noted patients whose tumors harbor a 1p19q co-deletion benefit particularly from the addition of procarbazine/lomustine/vincristine (PCV) chemotherapy to radiation therapy [25]. Likewise, another study of anaplastic oligodendrogliomas and oligoastrocytomas found that treatment (for progression free survival only), younger age, confirmed absence of residual tumor on imaging, frontal location, good WHO performance status, absence of endothelial abnormalities and/or necrosis, 1p19q co-deletion and IDH1 mutation were independent factors that predicted better progression free survival and overall survival [26].

IDH1 mutations have been reported in 68\% - 82\% of oligodendrogliomas [3] [5]. In our limited testing, IDH1 mutations were also slightly more common in the frontal lobe and superior frontal gyrus tumors, but again, not statistically significant. IDH mutation has been reported to be more common in isolated insular low grade gliomas than in paralimbic tumors [27]. Unlike 1p19q prognostic data, IDH1 mutation is linked to improved overall survival [5]. Although IDH1 mutation and 1p19q deletion often occur together, IDH1 mutation also seems to predict improved survival even without 1p19p deletion [28] [29]. Neither IDH1 nor 1p19q co-deletion can be definitively stated to be predictive of response to therapy [5].

Study weaknesses are those inherent to a retrospective study; loss of patients to follow up, changing management strategies over the years, and the lack of molecular data on patients diagnosed before tests were available. However, the large number of patients and the long duration of follow up make these data valuable. As low grade oligodendrogliomas are rare and slow growing, the results reported herein will be useful in combination with other centers' experiences in future meta-analyses.

\section{Conclusion}

Low grade oligodendrogliomas tend to occur more frequently in the frontal lobe, and particularly in the SFG, than can be accounted for by brain volume alone. 1p19q co-deletion and IDH1 mutation did not appear to be more common in frontal lobe tumors than those in extra-frontal locations. This differs from previous reports. There did not appear to be any advantage in recurrence rate, progression free survival, or life expectancy for 
tumors in the frontal lobe or in the SFG. 1p19q co-deletion did not appear to confer an advantage as measured by time to recurrence, time to progression, or overall survival. Other than age, eloquent location, Karnofsky status, and overall tumor size [17], widely available molecular markers for overall survival in low grade oligodendrogliomas are not currently available.

\section{Acknowledgements}

The authors thank Shirley McCartney, Ph.D., for editorial assistance.

\section{Conflicts of Interest}

There are no conflicts of interest to declare.

\section{References}

[1] Ross, D., Yang, L., Sagher, O. and Ross, A. (2011) Radical Resection of Adult Low Grade Oligodendroglioma without Adjuvant Therapy: Results of a Prospective Treatment Protocol-Surgical Treatment of Low-Grade Oligodendroglioma. Journal of Cancer Therapy, 2, 235-242. http://dx.doi.org/10.4236/jct.2011.22030

[2] Louis, D., Ohgaki, H., Wiestler, O. and Cavenee, W. (2007) World Health Organization Classification of Tumours. Pathology and Genetics of Tumours of the Nervous System. WHO Press, Lyon.

[3] Okamoto, Y., Di Patre, P.L., Burkhard, C., Horstmann, S., Jourde, B., Fahey, M., et al. (2004) Population-Based Study on Incidence, Survival Rates, and Genetic Alterations of Low-Grade Diffuse Astrocytomas and Oligodendrogliomas. Acta Neuropathologica, 108, 49-56. http://dx.doi.org/10.1007/s00401-004-0861-z

[4] Alentorn, A., Sanson, M. and Idbaih, A. (2012) Oligodendrogliomas: New Insights from the Genetics and Perspectives. Current Opinion in Oncology, 24, 687-693. http://dx.doi.org/10.1097/CCO.0b013e328357f4ea

[5] Bourne, T.D. and Schiff, D. (2010) Update on Molecular Findings, Management and Outcome in Low-Grade Gliomas. Nature Reviews Neurology, 6, 695-701. http://dx.doi.org/10.1038/nrneurol.2010.159

[6] Rodriguez, F.J. and Giannini, C. (2010) Oligodendroglial Tumors: Diagnostic and Molecular Pathology. Seminars in Diagnostic Pathology, 27, 136-145. http://dx.doi.org/10.1053/j.semdp.2010.05.001

[7] Sanai, N., Chang, S. and Berger, M.S. (2011) Low-Grade Gliomas in Adults. Journal of Neurosurgery, 115, $948-965$. http://dx.doi.org/10.3171/2011.7.JNS101238

[8] van den Bent, M.J., Snijders, T.J. and Bromberg, J.E. (2012) Current Treatment of Low Grade Gliomas. Memo, 5, 223-227. http://dx.doi.org/10.1007/s12254-012-0014-3

[9] Chang, E.F., Clark, A., Smith, J.S., Polley, M.Y., Chang, S.M., Barbaro, N.M., et al. (2011) Functional MappingGuided Resection of Low-Grade Gliomas in Eloquent Areas of the Brain: Improvement of Long-Term Survival. Journal of Neurosurgery, 114, 566-573. http://dx.doi.org/10.3171/2010.6.JNS091246

[10] Snyder, L.A., Wolf, A.B., Oppenlander, M.E., Bina, R., Wilson, J.R., Ashby, L., et al. (2014) The Impact of Extent of Resection on Malignant Transformation of Pure Oligodendrogliomas. Journal of Neurosurgery, 120, 309-314. http://dx.doi.org/10.3171/2013.10.JNS13368

[11] Capelle, L., Fontaine, D., Mandonnet, E., Taillandier, L., Golmard, J.L., Bauchet, L., et al. (2013) Spontaneous and Therapeutic Prognostic Factors in Adult Hemispheric World Health Organization Grade II Gliomas: A Series of 1097 Cases: Clinical Article. Journal of Neurosurgery, 118, 1157-1168. http://dx.doi.org/10.3171/2013.1.JNS121

[12] Marko, N.F. and Weil, R.J. (2013) The Molecular Biology of WHO Grade II Gliomas. Neurosurgical Focus, 34, E1. http://dx.doi.org/10.3171/2012.12.FOCUS12283

[13] Riemenschneider, M.J. and Reifenberger, G. (2010) Molecular Neuropathology of Low-Grade Gliomas and Its Clinical Impact. Advances and Technical Standards in Neurosurgery, 35, 35-64. http://dx.doi.org/10.1007/978-3-211-99481-8_2

[14] Brain Health and Puzzles (2012) Fun Facts about the Brain. http://www.brainhealthandpuzzles.com/fun facts about the brain.html

[15] El-Hateer, H., Souhami, L., Roberge, D., Maestro, R.D., Leblanc, R., Eldebawy, E., et al. (2009) Low-Grade Oligodendroglioma: An Indolent but Incurable Disease? Journal of Neurosurgery, 111, 265-271. http://dx.doi.org/10.3171/2008.11.JNS08983

[16] Zlatescu, M.C., Tehrani Yazdi, A., Sasaki, H., Megyesi, J.F., Betensky, R.A., Louis, D.N. and Cairncross, J.G. (2001) Tumor Location and Growth Pattern Correlate with Genetic Signature in Oligodendroglial Neoplasms. Cancer Research, 61, 6713-6715. 
[17] Chang, E.F., Smith, J.S., Chang, S.M., Lamborn, K.R., Prados, M.D., Butowski, N., et al. (2008) Preoperative Prognostic Classification System for Hemispheric Low-Grade Gliomas in Adults. Journal of Neurosurgery, 109, 817-824. http://dx.doi.org/10.3171/JNS/2008/109/11/0817

[18] Thon, N., Eigenbrod, S., Grasbon-Frodl, E.M., Ruiter, M., Mehrkens, J.H., Kreth, S., Tonn, J.C., Kretzschmar, H.A. and Kreth, F. (2009) Novel Molecular Stereotactic Biopsy Procedures Reveal Intratumoral Homogeneity of Loss of Heterozygosity of 1p/19q and TP53 Mutations in World Health Organization Grade II Gliomas. Journal of Neuropathology \& Experimental Neurology, 68, 1219-1228. http://dx.doi.org/10.1097/NEN.0b013e3181bee1f1

[19] Megyesi, J.F., Kachur, E., Lee, D.H., Zlatescu, M.C., Betensky, R.A., Forsyth, P.A., et al. (2004) Imaging Correlates of Molecular Signatures in Oligodendrogliomas. Clinical Cancer Research, 10, 4303-4306. http://dx.doi.org/10.1158/1078-0432.CCR-04-0209

[20] Jenkinson, M.D., du Plessis, D.G., Smith, T.S., Joyce, K.A., Warnke, P.C. and Walker, C. (2006) Histological Growth Patterns and Genotype in Oligodendroglial Tumours: Correlation with MRI Features. Brain, 129, 1884-1891. http://dx.doi.org/10.1093/brain/awl108

[21] Brown, R., Zlatescu, M., Sijben, A., Roldan, G., Easaw, J., Forsyth, P., et al. (2008) The Use of Magnetic Resonance Imaging to Noninvasively Detect Genetic Signatures in Oligodendroglioma. Clinical Cancer Research, 14, $2357-2362$. http://dx.doi.org/10.1158/1078-0432.CCR-07-1964

[22] Capelle, L., Oei, P., Teoh, H., Hamilton, D., Palmer, D., Low, I. and Campbell, G. (2009) Retrospective Review of Prognostic Factors, Including 1p19q Deletion, in Low-Grade Oligodendrogliomas and a Review of Recent Published Works. Journal of Medical Imaging and Radiation Oncology, 53, 305-309. http://dx.doi.org/10.1111/j.1754-9485.2009.02074.x

[23] Kesari, S., Schiff, D., Drappatz, J., LaFrankie, D., Doherty, L., Macklin, E.A., et al. (2009) Phase II Study of Protracted Daily Temozolomide for Low-Grade Gliomas in Adults. Clinical Cancer Research, 15, 330-337. http://dx.doi.org/10.1158/1078-0432.CCR-08-0888

[24] Tosoni, A., Franceschi, E., Ermani, M., Bertorelle, R., Bonaldi, L., Blatt, V. and Brandes, A.A. (2008) Temozolomide Three Weeks on and One Week off as First Line Therapy for Patients with Recurrent or Progressive Low Grade Gliomas. Journal of Neuro-Oncology, 89, 179-185. http://dx.doi.org/10.1007/s11060-008-9600-y

[25] Roth, P., Wick, W. and Weller, M. (2013) Anaplastic Oligodendroglioma: A New Treatment Paradigm and Current Controversies. Current Treatment Options in Oncology, 14, 505-513. http://dx.doi.org/10.1007/s11864-013-0251-7

[26] Gorlia, T., Delattre, J.Y., Brandes, A.A., Kros, J.M., Taphoorn, M.J., Kouwenhoven, M.C., et al. (2013) New Clinical, Pathological and Molecular Prognostic Models and Calculators in Patients with Locally Diagnosed Anaplastic Oligodendroglioma or Oligoastrocytoma. A Prognostic Factor Analysis of European Organisation for Research and Treatment of Cancer Brain Tumour Group Study 26951. European Journal of Cancer, 49, 3477-3485. http://dx.doi.org/10.1016/j.ejca.2013.06.039

[27] Goze, C., Mansour, L., Rigau, V. and Duffau, H. (2013) Distinct IDH1/IDH2 Mutation Profiles in Purely Insular versus Paralimbic WHO Grade II Gliomas. Journal of Neurosurgery, 118, 866-872. http://dx.doi.org/10.3171/2012.11.JNS121100

[28] Dubbink, H.J., Taal, W., van Marion, R., Kros, J.M., van Heuvel, I., Bromberg, J.E., et al. (2009) IDH1 Mutations in Low-Grade Astrocytomas Predict Survival but Not Response to Temozolomide. Neurology, 73, 1792-1795. http://dx.doi.org/10.1212/WNL.0b013e3181c34ace

[29] Labussiere, M., Idbaih, A., Wang, X.W., Marie, Y., Boisselier, B., Falet, C., et al. (2010) All the 1p19q Codeleted Gliomas Are Mutated on IDH1 or IDH2. Neurology, 74, 1886-1890.

http://dx.doi.org/10.1212/WNL.0b013e3181e1cf3a 
Scientific Research Publishing (SCIRP) is one of the largest Open Access journal publishers. It is currently publishing more than 200 open access, online, peer-reviewed journals covering a wide range of academic disciplines. SCIRP serves the worldwide academic communities and contributes to the progress and application of science with its publication.

Other selected journals from SCIRP are listed as below. Submit your manuscript to us via either submit@scirp.org or Online Submission Portal.
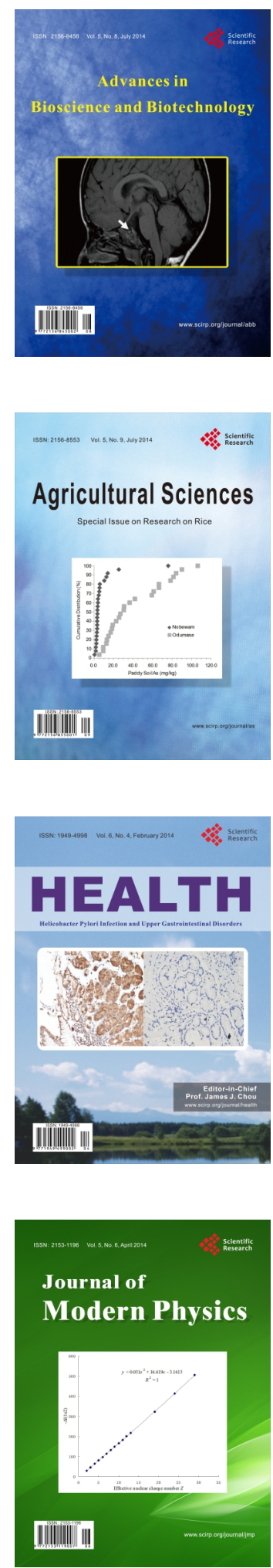
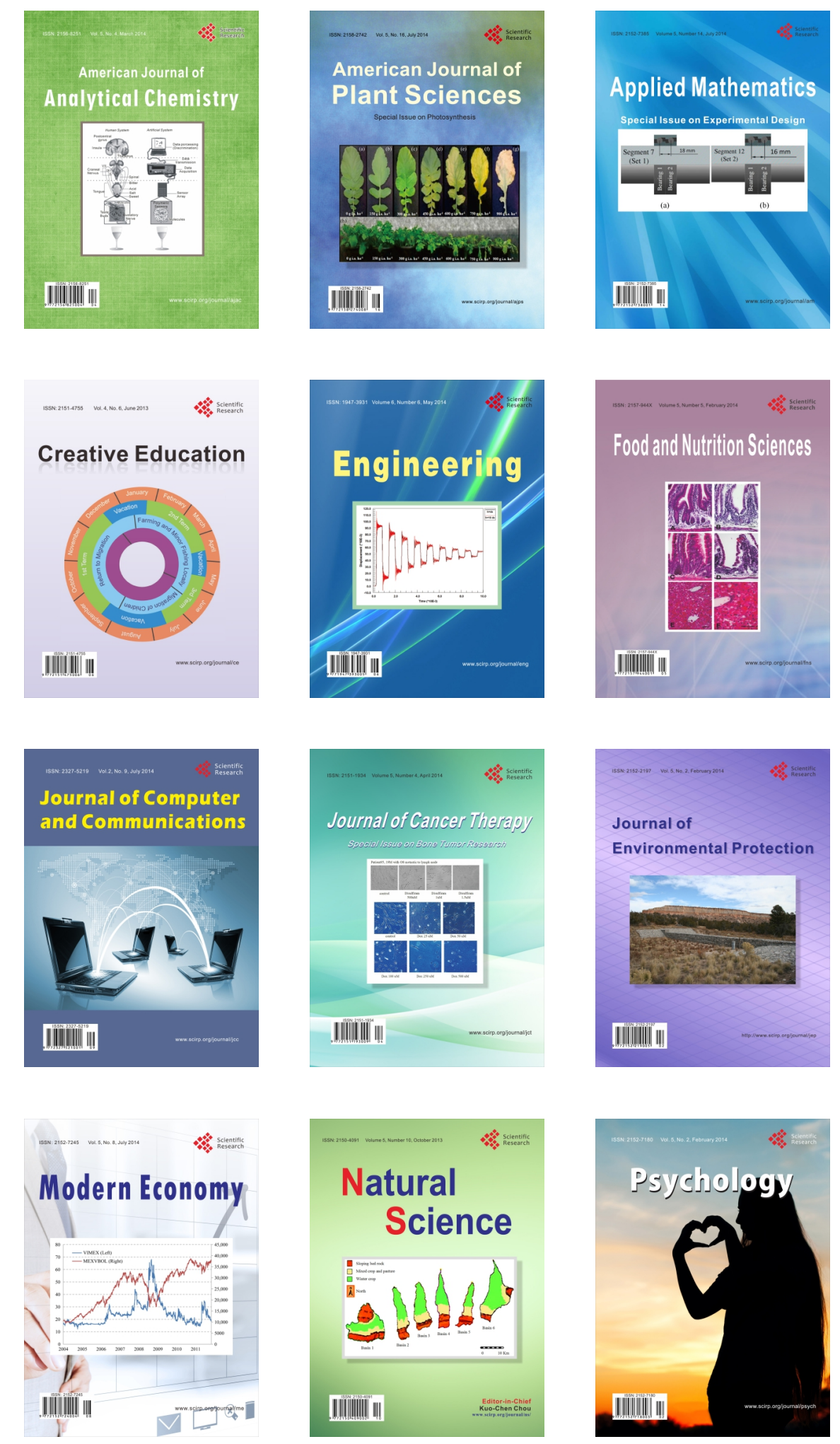